\title{
Context-aware BPM Using IoT-integrated Context Ontologies and IoT-enhanced Decision Models
}

\author{
Rongjia Song \\ Department of Information \\ Management \\ Beijing Jiaotong University \& $K U$ \\ Leuven \\ Beijing, China \& Leuven, Belgium \\ rongjia.song@kuleuven.be \\ rjsong@bjtu.edu.cn \\ Ying Wang \\ Department of Information \\ Management \\ Beijing Jiaotong University \\ Beijing, China \\ ywang1@bjtu.edu.cn
}

\author{
Jan Vanthienen \\ Department of Decision Sciences and \\ Information Management \\ KU Leuven \\ Leuven, Belgium \\ jan.vanthienen@kuleuven.be
}

\author{
Weiping Cui \\ State Grid Energy Research Institute \\ State Grid Corporation of China \\ Beijing, China \\ cuiweiping@sgeri.sgcc.com.cn
}

\begin{abstract}
IoT (Internet of Things) has the capability of capturing dynamic context from the physical world into the digital world. Context-aware BPM (Business Process Management) should integrate IoT as a key perspective of dynamic context of a business process and to enhance the decision making in a business process. IoT is often used to automate the process execution or integrated in the process model as resources of smart devices and additional concepts. In this way, IoT data is directly used without processing or reasoning with other contextual data to obtain higher-order contextual knowledge, which impairs its potential capability. The context layer and the decision layer are still missing while integrating IoT in BPM to obtain context-awareness. Decisions are still considered within context-aware BPM in a traditional way. This paper provides a separate concern of decisions from the process flow. We propose that the context-aware BPM ecosystem consists of four components which are: context-aware process models, context models, decision models and contextaware process execution. A framework is proposed to connect the IoT infrastructure to the context-aware BPM ecosystem using IoT-integrated ontologies and IoT-enhanced decision models, which enables the capabilities of IoT to make business processes and the decision making involved aware of the dynamic context.
\end{abstract}

Keywords-Context-aware, Business Process Management, IoT, Decision model, Context model

\section{INTRODUCTION}

IoT promises to create a world where all the objects around us are connected to the Internet. This allows people and things to be connected anytime, anyplace, with anything and anyone, ideally communicating with each other with minimum human intervention [1]. Recent studies also indicate BPM (Business Process Management) can benefit from IoT [2] and IoT is commonly used to automate and improve the operational efficiency in BPM, especially for process reengineering [3].

Since IoT has the capability of capturing dynamic context from the physical world into the digital world, contextual information that is invisibly embedded in the diverse environment and system behavior can be continuously and automatically collected by the IoT infrastructure. IoT concepts or devices are integrated in business processes as a resource in some studies [4-6]. However, IoT integration in the context management of a business process is still limited since there is a lack of a methodological framework to connect IoT infrustructure to BPM. More specifically, the context of a business process needs IoT data as a dynamic perspective, and decisions of a business process need IoT data to be agile. Then the research question can be presented as "how to bridge the IoT infrustructure to business processses for obtaining context-awareness?".

IoT devices continuously generate enormous amounts of up-to-date data, which is the key data source for dynamic data analytics. Hence, IoT coupled to intelligent data analytics, is the cornerstone technology to make better-informed decisions. Although IoT data is often used for making agile decisions in the ubiquitous and pervasive computing paradigm such as smart home applications, it is still challenging to utilize IoT to enhance the decision dimension of business processes in a methodological way. An important reason is that decisions are traditionally hardcoded inside business processes which impairs the capability of decisions and data analytics in business processes since the added value of data analytics lies in improving decision making of a process.

Recently, BPM is moving towards the separation of concerns paradigm by externalizing the decisions from the process flow. Embedding decisions in processes may increase complexity and diminish maintainability. Most notably, the introduction of the DMN (Decision Model and Notation) standard [7] provides a solution and technique to model decisions and the process separately but consistently integrated, which reduce the complexity of process flow and meanwhile formalize the decision making. However, in the literature on context-awareness in BPM, decisions are still considered inside processes in a traditional way. Hence, we separate the concern of decisions from the process flow and propose a framework to bridge the gap of IoT infrastructure and context-aware BPM using IoT-enhanced decision models. The ontology technique is also used to model the context of a business process with IoT data integrated for the dynamism.

This paper is constructed as follows. The related work is presented in Section II. Section III examines the role of 
decisions in context-aware BPM, and the ecosystem of context-aware BPM is presented in Section IV. Section V proposes the framework to connect the IoT infrastructure to context-aware BPM by integrating IoT data into the context of a business process and to enhance decision models of a business process, and a representative case is presented in Section VI. The benefits and limitations of applying the proposed framework are discussed in Section VII. In Section VIII, the conclusion and future perspectives are presented.

\section{RELATED WORK}

Using IoT to optimize BPM in existing research mostly focuses on automating the process execution and improve the performance efficiency by IoT-motivated process redesign or integrating IoT devices or concepts in the business process as a resource. For instance, in [3], a case study of the business process redesign based on the IoT technique in a harbour is presented to depict how the IoT infrastructure impacts the process design and how the process performance benefits from IoT-motivated process redesign.

In terms of IoT-integrated process modeling, several IoTrelated concepts have been identified, abstracted and expressed (i.e. IoT task, device, service, resource, acting user, etc.) for mapping to the BPM concepts, which are then integrated into process modeling as a key source of contextual information from entity-based or resource-oriented perspectives [4, 5, 8]. Research [1] indicates the semantic hierarchies of contextual data in the IoT paradigm, i.e., contextual information may have a different level of granularity. However, existing solutions directly integrate IoT entities into business processes as resources, which is not able to support the semantic representation of massive IoT data on context reasoning, data analytics or decision making. Therefore, the context layer and decision layer are still missing to obtain the added value of IoT awareness in BPM. Some other studies provide a solution of integrating IoT into context modeling or knowledge base such as in $[1,9,10]$. The ontology technique is proposed as one of the best ways for context modeling because of its expressiveness of formalization and capability of inference $[1,11]$.

IoT data is also used in data analytics to improve decision making in some studies. For instance, an intelligent model for logistics management based on geofencing algorithms and RFID technology to detect inconsistency and respond at realtime in [12]. Nevertheless, there is still a lack of a methodological way to utilize IoT data in the decision dimension of business processes and also bring the data analytics capability in decision making in the BPM area, since decisions are considered within context-aware business processes in a traditional way, which means that they are not modelled separately, but are hardcoded in the process model.

$\mathrm{DMN}$ is designed as a declarative decision language to model the decision dimension of business processes, which consists of two levels that are to be used in conjunction [7]. One is the decision requirements level, represented by DRDs (Decision Requirements Diagrams), which depicts requirements and dependencies between data and subdecisions involved in the decision model. Figure 7 depicts examples of a DRD. The input data can be static data or dynamic data, and may be extracted directly from databases, sensors and IoT devices, or generated by data fusion, data analytics and machine learning. The other level is the decision logic level, which can be represented by BKMs (Business
Knowledge Models) which encapsulate business know-how in the form of decision rules or a decision table.

As illustrated above, we observed that some researchers have noted the impact of the IoT paradigm on BPM, contextaware computing and decision making respectively. However, business processes, context and decisions are components in the ecosystem of context-aware BPM. Hence, it is of vital importance to construct a framework to connect the IoT infrastructure to context-aware BPM using IoT-integrated context models and IoT-enhanced decision models, which is also the way to bring the capability of dynamic data analytics in the BPM optimization.

\section{ON THE ROLE OF DECISIONS IN CONTEXT-AWARE BPM}

Context affects business processes both at process design time and run time. At design time, context is considered to optimize process models for responding to different requirements of situations and customers. At run time, context is integrated to execute process correctly and automatically.

Decisions are traditionally considered within the business process. However, leading by the recent research trend [13], in case of separately considering decisions from the process flow, we can state that decisions play the key role in contextaware BPM. A detailed illustration is in [14]. Different types of decisions influence context-aware effects on business processes in their own way.

Context-awareness can bring a process flexibility, personalization and automation only if this process may change according to the dynamic context. Decisions are crucial stimuli that may trigger process changes in different situations. If various types of decisions may influence the business process changes on either process modeling or execution, this base process then needs context-awareness. On the basis of the stimuli, how the business process varies from different outcomes of decisions is important to anticipate process variants. Then the adaptation points and options can be anticipated by identifying how different outcomes of decisions are utilized in the business process.

Modeling or executing a context-aware business process needs to integrate the context in the base business process. So what contextual variables and data a business process needs is the next issue to solve, i.e., the contextualization of a business process. It cannot merely rely on expert knowledge which may lead to incompleteness or redundancy, especially in complicated situations. The data inputs of context-dependent decisions and their sub-decisions are the essential sources to identify relevant contextual variables of a business process.

\section{A. Strategic Decisions}

Some strategic decisions could lead to business process variants. For instance, with different levels of requirements on implementation costs, quality control, or safety inspection, business process models may vary according to different contexts. Context-awareness is an effective way to obtain flexibility by modeling context-dependent process variants [15], and the process invariant is designed to keep the "identity" of the base process [16]. Each process variant constitutes an adjustment of the base process according to requirements of the process context. Hence, contexts that could trigger the need of process variants, and the corresponding variants need to be anticipated. These possible contexts sentence from various effects of strategic decisions and contextual variables involved need to be identified. Then 
process variants need to configured with the base business process by integrating contextual data.

\section{B. Tactical Decisions}

Tactical decisions towards user satisfaction are essential to model context-aware business processes for providing personalized services. Recent advances in IT such as IoT and mobile devices provide ways to sense users' contexts. A usercentric paradigm of ubiquitous and pervasive computing provides users access to preferred services anytime [17]. Delivering personalized services motivates tactical decisions and considering context in the business process design [18]. Dynamic context and user preferences need to be considered in the process design to deliver preferred services with minimal user intervention.

\section{Operational Decisions}

Operational decisions are made rapidly and frequently during the process execution which controls or influences the organization's business behavior [19]. More specifically, a work around that is often observed in context-aware business process modeling practices is that a contextual variable becomes an explicit condition of a process leading to a decision point. Decisions points, of the operational type, involved in a business process could lead to gateways for handling different scenarios. In case that the decisions are context-dependent, dynamic context therefore needs to be identified for correctly executing one of the process branches. Multi-sources contextual data related is then needed to produce up-to-date knowledge for the context identification.

Some decision activities are context-dependent since contextual data is needed for the decision execution. Hence, context can affect process execution in decision activities by providing contextual data as parameters of their prevailing circumstances (inputs) for calculation. It does not lead to changes in flow splits of business processes, but the outcome can be incorrect without up-to-date context integrated.

\section{The ECOSystem of CONTEXT-AWARE BPM}

Addressing context in BPM aims to enable the business process to be aware of its context at both design time and run time for responding, reacting and adapting to dynamic situation correctly and automatically. The context of a business process therefore needs to be identified, integrated and managed in the whole life cycle of BPM. Moreover, decisions should be separately concerned from the process flow since decisions play a key role in context-aware BPM.

Considering the interconnection and interaction with the surroundings, context-aware BPM should be an ecosystem with the capabilities of self-organization, self-sustaining and co-evolvement, in order to obtain more ordered and better informed system embedded in dynamic situations.

- Self-organization: Context-aware BPM should be able to connect its components with minimum intervention from outside of the system.

- Self-sustaining: The system should be organized in an extendible way to enable its usability even in changing market or situations.

- Co-evolvement: The components of context-aware BPM system should be able to adapt when any of them changes based on their interdependency.

The business process ecosystem was traditionally proposed in [20] which consists of business processes, business users, IT developers, software modules, since there is mutual adaptation between these four components. In other words, change in one component will likely lead to reciprocal change in the other components.

As context-awareness is accommodating to the business process, context is considered as all relevant information to

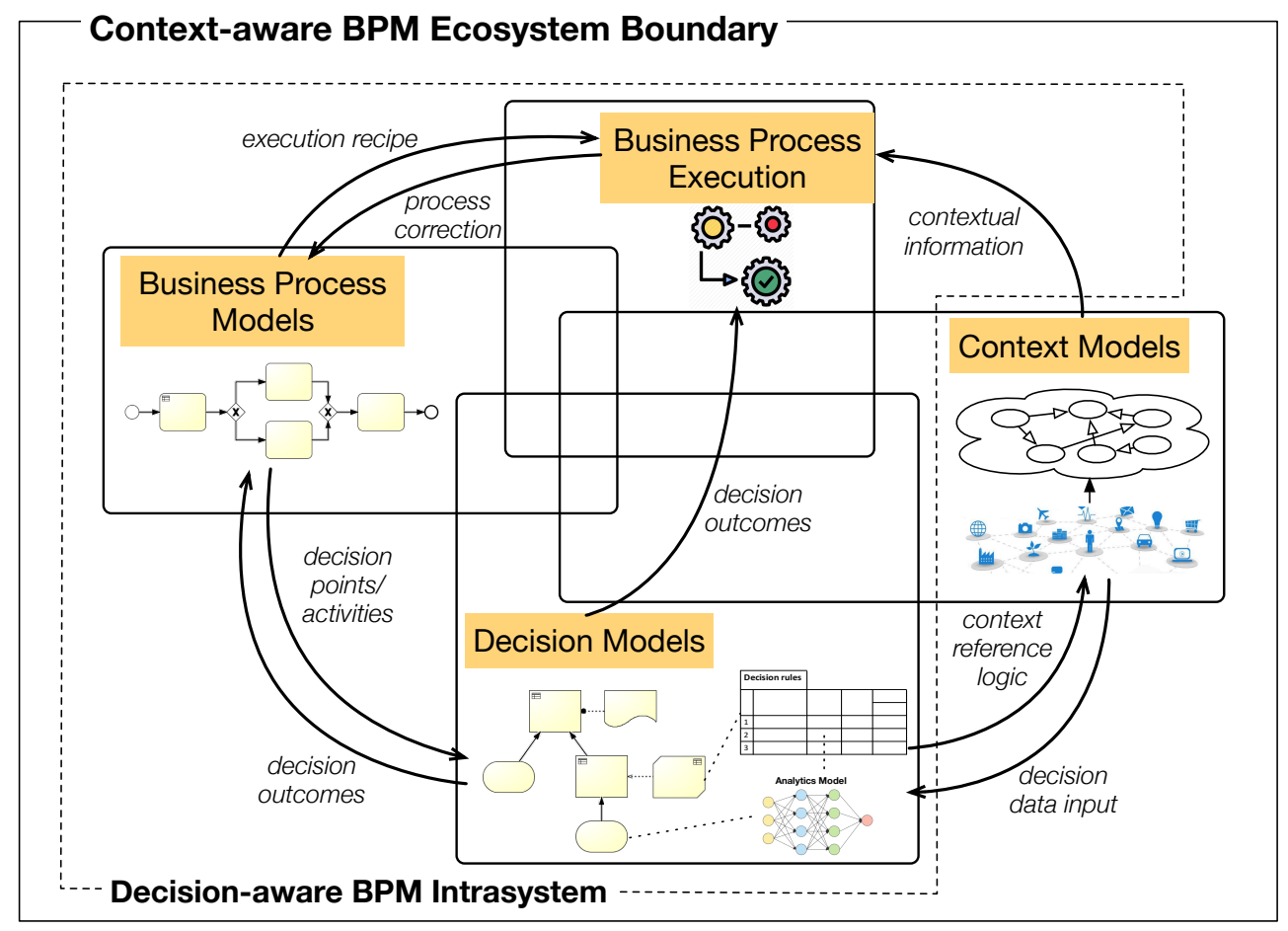

Figure 1 The context-aware BPM ecosystem 
characterize the situation of a business process including users, systems and their interaction [21]. Hence, business users, IT developers, software modules can be considered as different components of the context concept. Moreover, if decisions are still hardcoded in context-aware BPM, decisions' capability will be impaired. Thus, decision models need to be externalized from business process models, which include dependencies of sub-decisions and data requirements, as well as BKMs expressing the decision logic. Contextual data is one of the key sources for agile and informed decision making according to dynamic situations. Decisions can therefore be considered as the connection of business processes and its context. The context model is needed to organize contextual data and its relations in an extendible and usable way, especially if the situation in which the business process embeds is complicated. In particular, IoT devices are emerging data sources of system behaviors and external environments for dynamic data analytics to obtain the contextawareness of business processes. Furthermore, context is not only needed for designing process models, but also for the process execution. Contextual information needs to be distributed to the appropriate point or person in time for supporting the correct process execution. Hence, business process execution should also be considered as a component of the context-aware BPM ecosystem.

To develop a model of the context-aware BPM ecosystem, we state that there is mutual adaptation between the business process, the content of which we define to be process models at design time and process execution at run time, and its context, the content of which we define to be the context model, as well as decision models involved in the business process. These four components form the co-evolutionary core of the context-aware BPM ecosystem (Figure 1), in which context-dependent decisions bridge the gap between the business process and its context, as well as the decision making and contextual data including IoT data.

\section{A. Business Process Models}

A business process is a set of logically related tasks performed to achieve a defined business outcome with a logical organization of people, resources and procedures involved in work activities designed.

Business process models are designed as the recipe for process execution. In turn, process execution may reflect the impropriate process design for process correction or optimization, such as deadlocks in the process model.

\section{B. Decision Models}

Decision models document and formalize decision making such that decisions can be made consistently, verifiably and automated where appropriate. Decision models consist of the decision requirement level and the decision logic level. The decision model is the recipe for executing the decision, which helps exclude rules from business processes and provide decision outcomes as edge conditions of process gateways. In turn, gateways of a business process are commonly caused by decisions. Hence, process gateways indicates decision points and decision activities leading to the need of modeling decisions. Moreover, activities of a business process related to calculations are also considered as decision activities.

\section{Context Models}

Context models are used to organize and represent all relevant contextual entities and their relations in an extendible and usable way. Especially in the IoT paradigm, context is sensed from a variety of sources including physical sensors (i.e., IoT devices) and virtual sensors (i.e., applications and information systems), which differ in the quality, format and capture frequency [1]. Therefore, multi-sources contextual data may need a context model to organize, analyze and utilize for acquiring understandable contextual knowledge and further IoT-awareness.

As a sensor can only separately sense a piece of context, raw data can be meaningless without other information of interest. Hence, sensor fusion and context reasoning may be needed for the abstraction of contextual data. Moreover, context reference rules must be designed consistently with business logic, decision logic or general facts to ensure the correctness of context interpretation. These upper level contextual information can then be used as the data input of dynamic analytics and context-dependent decisions.

\section{Business Process Execution}

Business process execution is the actual operation to produce business products or achieve business objectives based on predefined business process models. However, given a syntactically correct process sequence, errors can still occur during the process execution due to the incorrect dataflow. Process execution need to correctly respond to dynamic context. Hence, the right contextual data need to be distributed to the appropriate person or point in time for identifying current situation. Regarding to correctly execute process branches of gateways and activity calculation, decision outcomes are needed for the process execution.

In addition, business process models, decision models and the business process execution constitute the decision-aware BPM intrasystem. Nevertheless, if context is not taken into consideration at the process design time or run time, the business process model or process execution could be incorrect in certain situations. Hence, we consider the whole picture of context-aware BPM as an ecosystem.

\section{BRIDGE IOT INFrastruCTURE AND CONTEXT-AWARE BPM ECOSYSTEM}

IoT coupled to intelligent data analytics, is the preeminent cornerstone technology for aligning decision-making and business processes to data, that could help to make betterinformed and context-aware decisions. IoT devices map physical objects to the digital world, which are emerging data sources of system behaviors and external environments for dynamic data analytics to obtain the context-awareness of business processes. However, there is a lack of a systematic framework to align IoT as an emerging technology and context-aware BPM.

Organizations are increasingly investing in data-driven analytics to improve their business results, deepen customer understanding and better risk management. The value of these analytics lies in improving decision-making. In other words, unless a decision is improved as a result of analytics it is hard to argue that the analytics have any value [7]. The DMN technique supports the ability to extract and operationalize value from data analytics since the value of data analytics lies in improving decision-making. Hence, it is of vital importance to use IoT data integrated in context models to enhance decisions in business processes and to obtain contextawareness in BPM. A framework to bridge context-aware BPM ecosystem and IoT Infrastructure is depicted in Figure 2. 


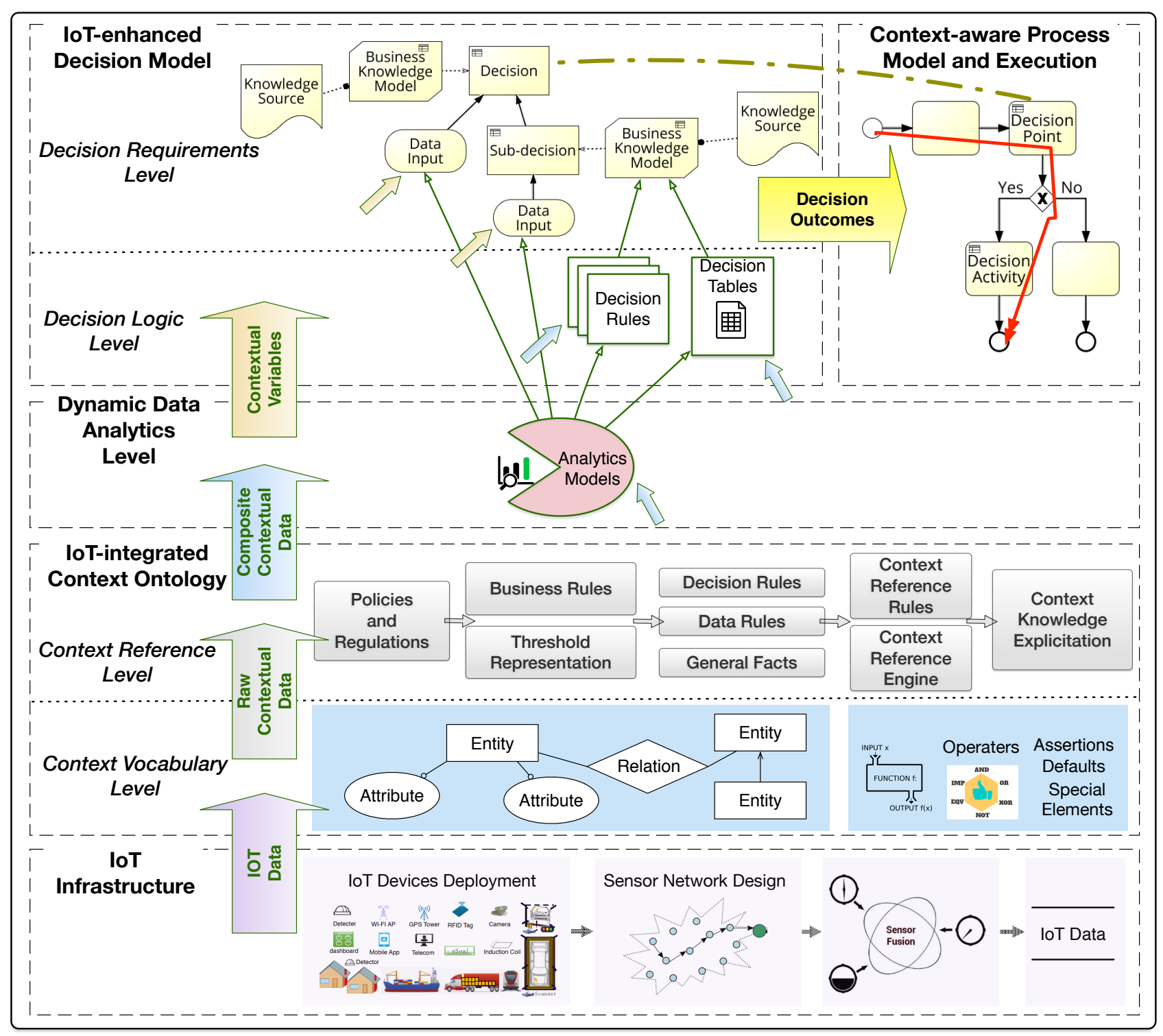

Figure 2 A framework to bridge context-aware BPM ecosystem and IoT Infrastructure

\section{A. IoT Infrastructure}

The application of various IoT techniques (e.g., RFID, induction coils, video detection, sensors, mobile terminals) leads to a large number of IoT devices deployed. It senses the external environment and the process execution, in the meanwhile, generating enormous sensor data. In particular, sensor networks need to be designed since a large number of sensors work in various ways. Sensor fusion is further needed to support the better understandability and usability of IoT data. In other words, sensor data could be only machine readable such as binary-coded data, which cannot be directly understood and used by users. After preprocessing, IoT data should be generally understandable such as a coordinate that consists of a longitude and latitude.

\section{B. IoT-integrated Context Model Using the Ontology Technique}

The context model consists of two levels that are used in conjunction. One is the context vocabulary level, which includes the design of contextual entities and assertions to constitute the knowledge base of context. The other level is the context reference level, which is based on analyzing business rules to design context reference rules for context reasoning, abstraction and knowledge extraction.

Different techniques have been proposed up until now to model context. The most popular ones are key-value pairs, markup languages, graphical models, object-oriented models, logic-based models and ontology-based models [1,22], and an exhaustive comparison of these techniques can be found in [1]. Although all of these techniques have advantages and disadvantages, the ontology technique is one of the best choices to model context and is drawing more and more attention as a generic and explicit way to capture and specify context [23]. The context ontology is formal representation and explicit specification of context related concepts and relationship that hold among those concepts. It is used to capture and describe shared knowledge about a domain of interest. It mainly contains elements of a) classes to represent entities of domain, b) individuals to represent objects of classes, c) object properties to describe the relationship between individuals, d) datatype properties to describe data attributes that are the relationship between an individual and data values, e) restrictions to express total or partial rules, and f) assertions to illustrate predefined axioms. 
Context is captured from various sources including IoT devices, databases, applications and manual inputs. These contextual data can be raw sensor data, identifiable IoT data or composite data, which leads to semantic hierarchies. Multisources contextual data is therefore needed to be reasoned for producing up-to-date knowledge, in order to identify dynamic context. Figure 3 depicts the semantic hierarchies of the IoTintegrated context.

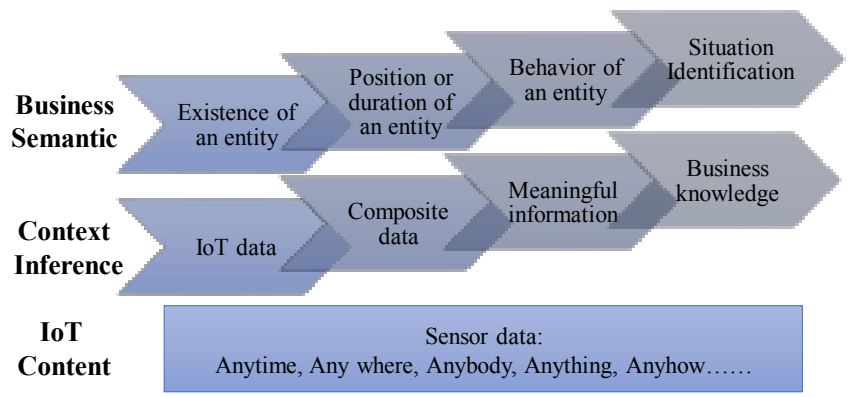

Figure 3 Semantic hierarchies in the IoT-integrated context

The vision for IoT is to bridge the gap between the physical world and its representation within the digital world, by the means of capturing the data are consumed and produced with respect to activities in business processes. Sensors and IoT devices automatically capture data as streams that contains the digital implication of external environment and system behavior, which should be considered as the key source of genuine and timely contextual data. The IoT content contains data related to any entities of interest (i. e., anything), and no matter anytime to sense, anywhere to locate, anybody to operate, and anyhow to access. Based on these IoT data, a hierarchical transformation of contextual inference is involved to raise the semantic level of business awareness.

A piece of raw sensor data can be meaningless especially regarding to the business objective. For instance, IoT data can be a GPS coordinate that consists of a longitude and a latitude, which is identifiable for the existence of an entity but not meaningful in terms of business purposes. This GPS coordinate considering the general facts of geography, composite data of country, city, building, floor, room and so on can be produced as the location to indicate the position of an entity. Furthermore, meaningful information can be extracted to examine the behavior of an entity such as determining if a staff is in the working position by comparing with the business logic. Finally, contextual information is used for reasoning to obtain useful business knowledge to identify situations or access business risks.

Reference rules are often needed for context reasoning to extract useful information. In order to ensure the correctness of context abstraction, context reference rules should be consistent with general facts or business rules including decision rules (i.e., disjunction of activities) and data rules (i.e., data constraints for activities occurrence). In other words, context interpretation should comply with the business logic. Moreover, the context reference engine is also needed for executing context reasoning. For instance, an example of context reference based on location information is depicted in Figure 4. This example involves several entities including 'staff', 'location', 'time' and 'working system'. 'outlook' is an individual of 'working system'. The entity 'staff' has some attributes including 'working or not' and 'working status'. The entity 'working system' has an attribute 'system status'.
Moreover, certain relations between entities also exist. An entity 'staff' accesses a 'working system', works at certain 'time' and is located in certain 'location'.

Ontologies guarantee a high degree of expressiveness, formality, and semantic richness, and exhibit prominent advantages for automatically inferring new knowledge about the current context [11]. Logical reasoning mechanisms based on formal rules are embedded in ontologies for the inference of new information based on lower-order raw data as well as to evaluate context by checking for business conformance. The ontology technique supports several inference engines embedded for semantic reasoning [22].

In addition, OWL (Web Ontology Language) is proposed as a machine-interpretable W3C (World Wide Web Consortium) standard that provides a semantic markup language designed to provide a vocabulary along with formal semantics to facilitate the management and processing of knowledge at run time. Moreover, SWRL rules can be used over OWL in order to infer new knowledge which are expressed in terms of ontology concepts (classes, properties and individuals).

\begin{tabular}{|c|c|c|c|}
\hline $\begin{array}{c}\text { Context } \\
\text { Inference } \\
\text { Layers }\end{array}$ & $\begin{array}{c}\text { Business } \\
\text { Semantic } \\
\text { Layers }\end{array}$ & $\begin{array}{c}\text { Conference } \\
\text { Reference Rules }\end{array}$ & Business Logic \\
\hline $\begin{array}{l}\text { regular, } \\
\text { overtime, } \\
\text { absent }\end{array}$ & $\begin{array}{l}\text { Working } \\
\text { status }\end{array}$ & $\begin{array}{l}\text { WorkingOrNot(?s, } \\
\text { "working") and } \\
\text { (Time(?t, [>=18]) } \\
\text { or Date(?d, } \\
\text { "isWeekend") => } \\
\text { WorkingStatus(?s, } \\
\text { "overtime") }\end{array}$ & $\begin{array}{l}\text { The working } \\
\text { status of a staff } \\
\text { can be } \\
\text { identified by } \\
\text { comparing the } \\
\text { current working } \\
\text { status with the } \\
\text { working agenda. }\end{array}$ \\
\hline $\begin{array}{l}\text { working, } \\
\text { not(work } \\
\text { ing) }\end{array}$ & $\begin{array}{l}\text { Working } \\
\text { or not }\end{array}$ & $\begin{array}{l}\text { Staff(?s) and } \\
\text { Outlook(?s, } \\
\text { "green") => } \\
\text { SystemStatus(?s, } \\
\text { "accessible") } \\
\text { Location(?s, } \\
\text { ?s.office) and } \\
\text { SystemStatus(?s, } \\
\text { "accessible") => } \\
\text { WorkingOrNot(?s, } \\
\text { "working") }\end{array}$ & $\begin{array}{l}\text { If a staff is in the } \\
\text { office and the } \\
\text { status of } \\
\text { working system } \\
\text { is accessible, the } \\
\text { staff is } \\
\text { considered as } \\
\text { working. }\end{array}$ \\
\hline $\begin{array}{l}\text { city, } \\
\text { building, } \\
\text { floor,....... }\end{array}$ & Location & \multicolumn{2}{|c|}{ Geographical facts } \\
\hline $\begin{array}{l}\text { longitude } \\
\text {, latitude }\end{array}$ & $\begin{array}{c}\text { Coordinat } \\
\text { e }\end{array}$ & \multicolumn{2}{|c|}{ Sensor fusion } \\
\hline C/A code & $\begin{array}{c}\text { GPS } \\
\text { signal }\end{array}$ & \multicolumn{2}{|c|}{ - } \\
\hline
\end{tabular}

Figure 4 Context reference based on location information

\section{IoT-enhanced Decision Model Using Dynamic Data Analytics}

After IoT data is integrated in the context of a business process, IoT should be also used to enhance the decision dimension of a business process. Decision models are the key to obtain added value of data analytics in business processes since the value of data analytics lies in improving decisionmaking. Dynamic data analytics relies on IoT data to provide the input of user environment and system behavior. 


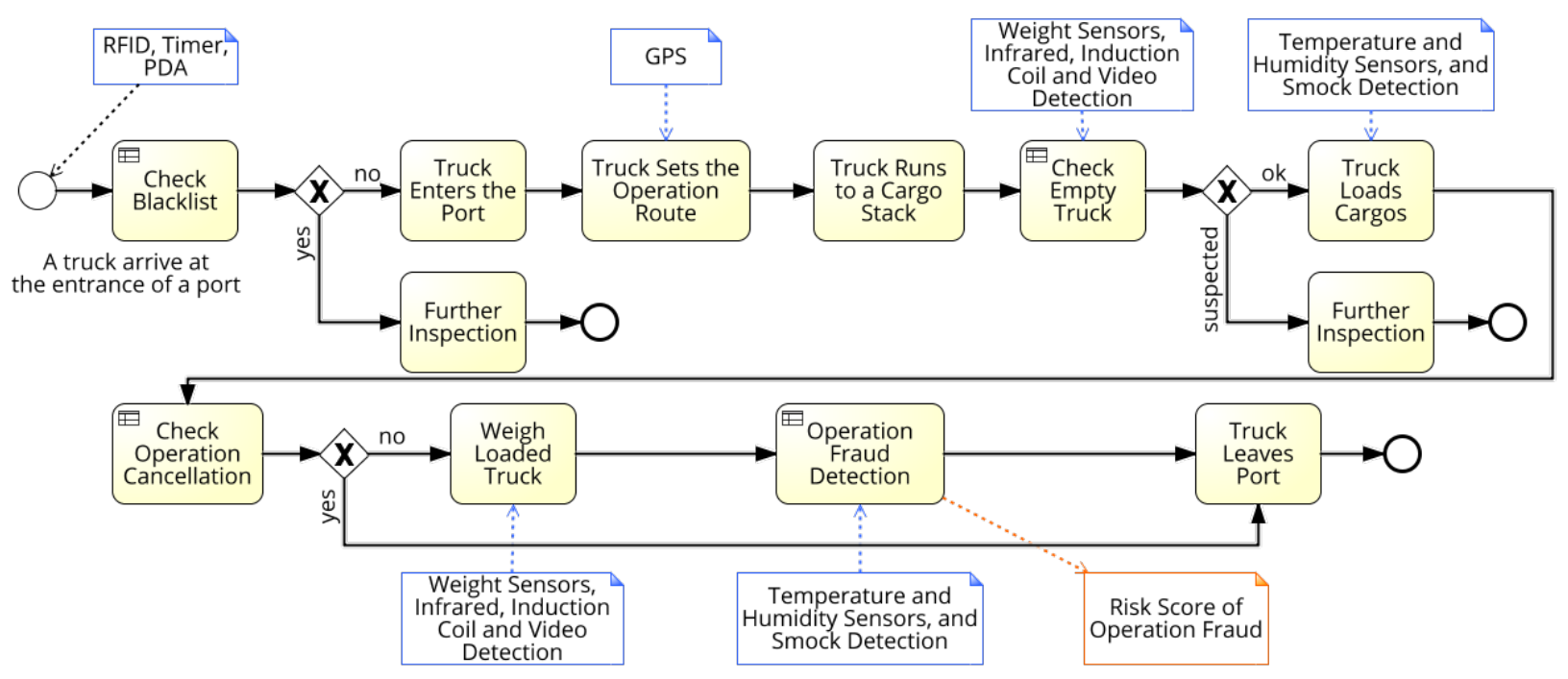

Figure 5 A representative business process of truck pick-up cargo in the IoT paradigm

IoT data is directly integrated in data analytics or business processes as a type of resource in a traditional way. However, IoT data is commonly raw data with relative low semantic level, which may be not able to be utilized in analytics or business process in many cases. Hence, the context model is a key layer to improve the understandability and capability of IoT data in data analytics and business processes. Dynamic analytics can also be an intermediate layer to make decisions with an awareness of the dynamic context. IoT data or IoTaware contextual data can also be used to directly improve decisions with a separate consideration of the decision dimension from the process flow by invoking contextual variables as parameters in decision rules or decision tables.

IoT integrated context ontologies can provide contextual knowledge as inputs of analytics models or decision requirements. Moreover, the outputs of analytics models can also support the input of business knowledge models, which can be decision rules or decision tables.

\section{Context-aware Business Processes}

After the IoT infrastructure is connected to context models, data analytics and decision models, the final step is to bridge decision models to business processes to complete this picture. BPM is moving towards the separation of concerns paradigm by externalizing decisions from the process flow. DMN provides a solution and technique to model decisions and the process separately but consistently integrated.

Decisions could lead to gateways and process variants in the phase of business process modeling. Different outcomes of decisions also affect the business process at run time, in the calculation of decision activities and execution of process branches. Hence, through the context layer and decision layer, IoT infrastructure finally connects to the business processes with the capability of dynamic data analytics integrated.

\section{A RePRESENTAtive CASE}

We take a business process of truck pick-up cargo as a representative case to depict how the proposed framework can be used in applications.

In order to make the business process aware of its context, the IoT infrastructure is constructed with various IoT devices deployed on important positions of the business process implementation in order to capture contextual data and automate the process. In this representative case, the following IoT devices are deployed for capturing the context of the truck pick-up cargo process.

- RFID is attached on the windshield of trucks, information of which can automatically be invoked during the process execution;

- GPS is activated after a truck enters the port for monitoring the location and route of a truck during the operation;

- Weight sensors, infrared, induction coils and video detection are deployed around weighbridges to make sure a truck is in the appropriate position for weighing;

- Temperature and humidity sensors as well as smoke detectors are deployed around cargo stacks and checking points to ensure the operation safety and cargo quality.

Figure 5 depicts the representative business process with IoT devices deployed (highlighted in the blue rectangles). In addition, several decisions including 'check blacklist', 'check empty truck', 'check operation cancellation' and 'operation fraud detection' are involved in this representative process. Some of context-aware decisions lead to splits of the business process, including decision activities of 'check blacklist', 'check empty truck' and 'check operation cancellation'. However, decision activities may not lead to gateways as well, such as 'operation fraud detection'. Contextual information is needed to calculate the outcome of the decision activity 'operation fraud detection'. Hence, the outcome 'risk score of operation fraud' can be incorrect without up-to-date context knowledge integrated.

An IoT-integrated ontological model is constructed for managing the context of the representative process, which is depicted as Figure 6. Seven top-level classes including 'truck', 'cargo', 'customer', 'geographical information', 'physical environment', 'time' and 'operation', as well as their subclasses, attributes and their relations constitute the context ontology. Explicating the relationship between contextual entities is important for organizing the context and context reasoning since classes, their individuals and their relations are used for expressing context reference rules. Then 


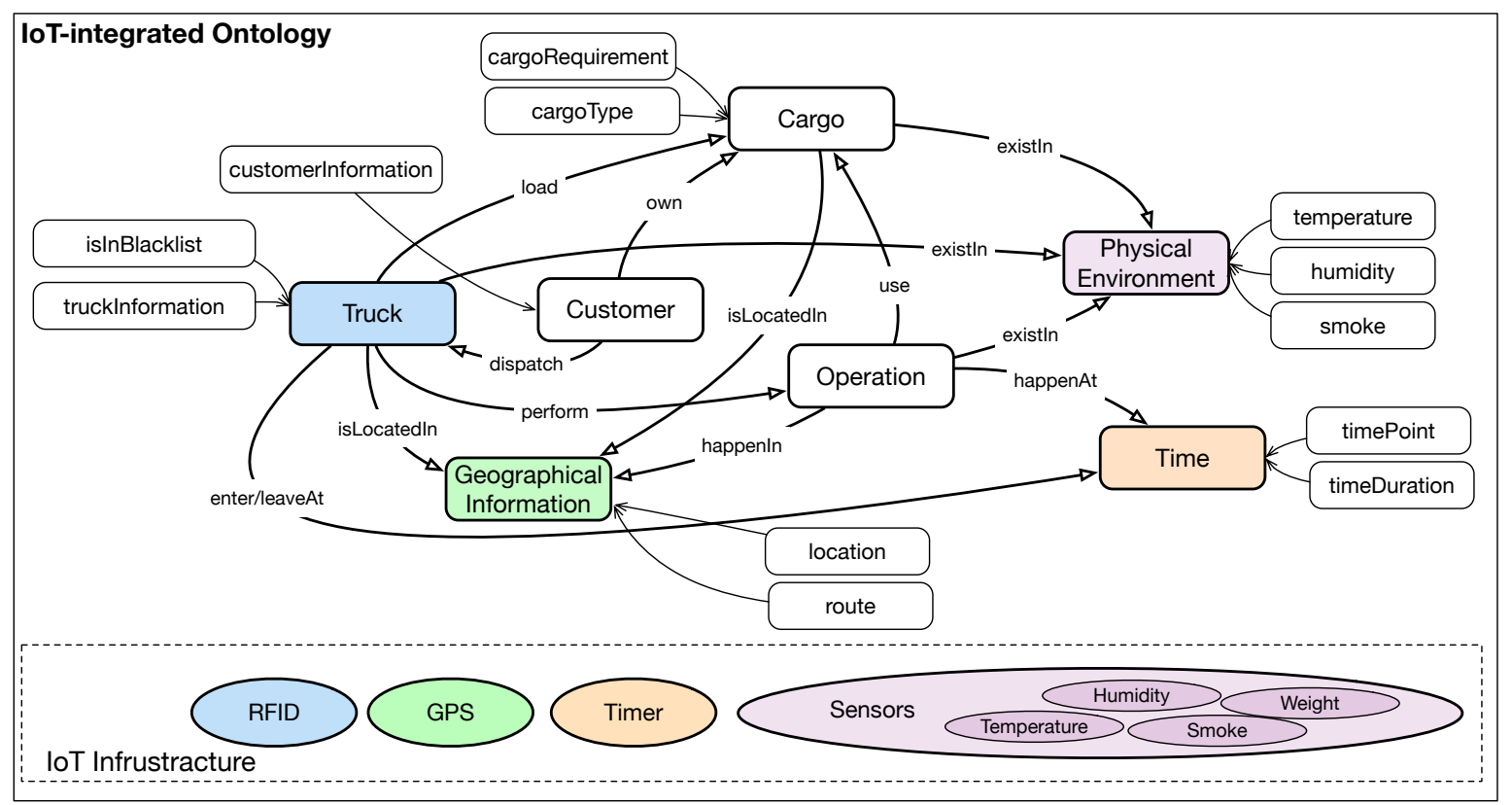

Figure 6 An ontological model of the context of the representative business process

contextual data can be reasoned to obtain high-order contextual information and knoweldge. The relationship between entities in the context ontology of this representative case are illustrated below.

- Trucks load Cargo; Trucks enter/leave at certain Time points; A Truck is located in certain Location; Trucks perform Operations; Trucks exist in certain Physical Environment;

- Cargo exist in certain Physical Environment; Cargo is located in certain Location;

- Customers own Cargo; Customers dispatch Trucks;

- Operations happen at certain Time points; Operations happen in certain Location; Operations use cargo; Operations exist in certain Physical Environment.
In particular, IoT data provide inputs or ingredients to produce the inputs for the context ontology.

- Truck VIN (Vehicle Identification Number) is automatically collected by RFID when a truck enters the port;

- The location and route monitoring are captured by the GPS;

- Physical environments including temperature, humidity and smoke are captured by sensors of multiple types, and the cargo weight is collected by weight sensors;

- Time points and duration are collected or calculated based on timers.
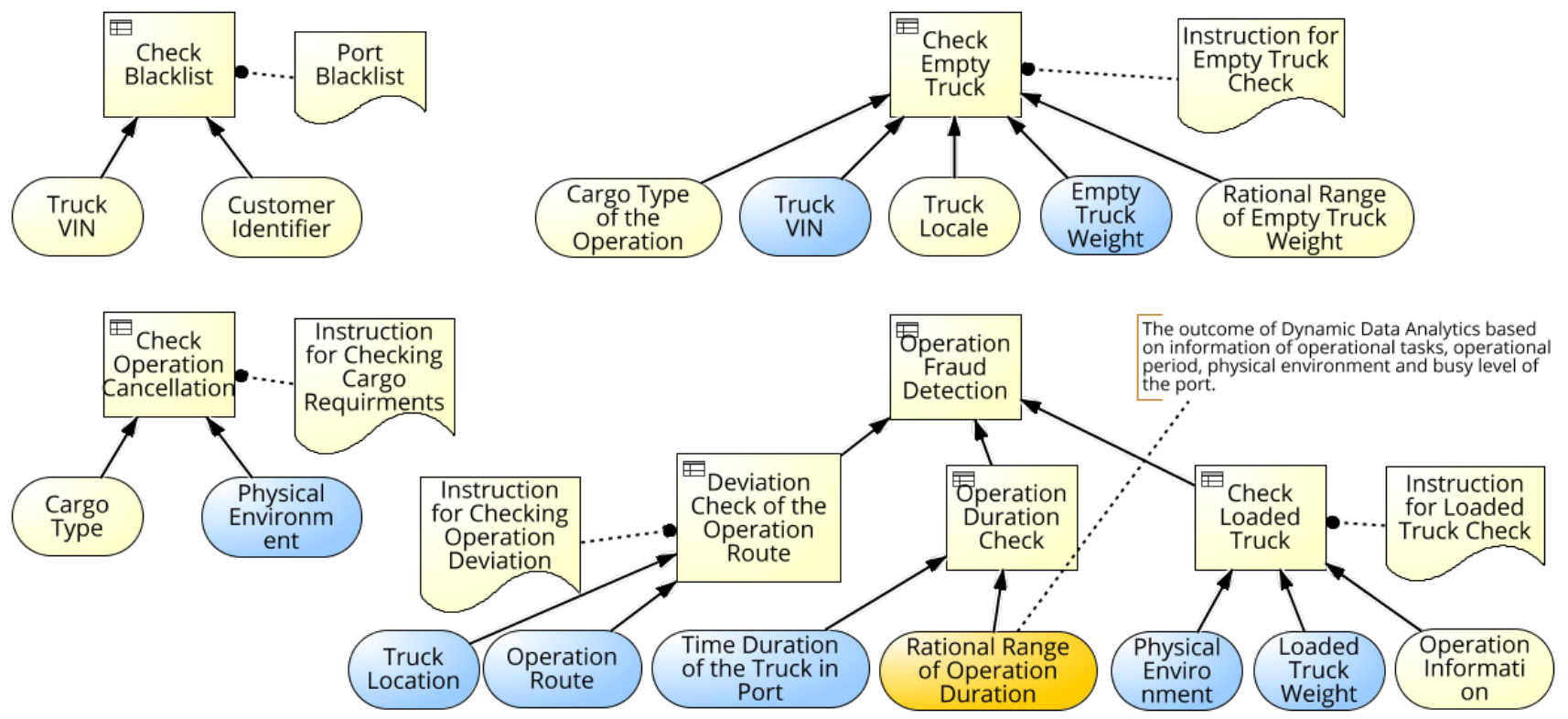

Figure 7 DRDs of the representative business process 
Besides contextual data that depend on IoT devices, some other information such as profilled information of operation, truck and cargo can be obtained by querying from databases. Hence, the ontological model sets the knowledge base for managing and reasoning the contextual knowledge of the business process.

After the context of the business process is determined, the decision dimension needs to be modeled to obtain IoTenhanced context-awareness in the decision making. Figure 7 depicts the DRDs of decisions in the representative business process. Some of these data requirements are contextdependent and part of them can be collected directly by IoT devices, such as truck weight from weight sensor, as well as truck location and route from GPS. Moreover, Truck VIN is an explicit input of this process which can also be directly collected from RFID, and other data needed including truck model, truck locale and so on can be extracted in terms of truck VIN. The input data of the DRDs depending on IoT devices is highlighted in the blue rounded rectangles in the Figure 7. Some other context-dependent decision requirements need to reason contextual data for obtaining contextual variables with higher semantic level. For instance, time duration of the truck in port needs to be calculated based on the entry time of truck and the current time.

Nevertheless, there are still some contextual variables which are still not clear what data constitute according to the DRDs, such as physical environment. Hence, decision logic needs to be further analyzed to identify contextual data involved as parameters in decision rules. For instance, if there is a decision rule related to the decision 'check operation cancellation':

If CargoType is "coal" and WeatherState is
"HeavyRain"
Then OperationCancellation is "yes"
Else if CargoType is "Coal" and
StackHumidity is True
Then OperationCancellation is "yes"
Otherwise OperationCancellation is "no"

Moreover, in order to determine the parameter "HeavyRain", the context reference rule is needed for context reasoning:

\section{Weather(?w) and Precipitation(?w, [>=25]) =>} WeatherState(?w, "HeaveyRain”)

According to this example of decision rule and reference rule, weather state and stack humidity can be identified as contextual variables, and precipitation can be determined as contextual data of lower semantic level.

In addition, decision 'operation duration check' needs to calculate between 'time duration of the truck in port' and 'rational range of operation duration' which is the outcome of dynamic data analytics. More specifically, the dynamic analytics of 'rational range of operation duration' is influenced by operational tasks, operational period, physical environment and busy level of the port.

In this way of applying the proposed framework, the IoT infrastructure effectively supports the supplement of processed IoT data as a key perspective of the context of a business process, dynamic inputs of data analytics and data requirements of decisions in order to enhance the decision making and context-awareness in BPM.

\section{DISCUSSION}

Context-aware BPM attracts more and more attention in academia and industries to obtain process flexibility and personalized services since the market is highly dynamic and diverse, especially in the ubiquitous and pervasive computing.

More specifically, contextual data, even including IoT data is used as a key source of inputs for analytics to improve decision making. The ontology technique is also commonly used for managing contextual information. Moreover, contextual data is also used to model and execute business process towards flexibility. However, existing research merely focus on one layer of the context-aware BPM system. There is a lack of a methodological framework to connect the IoT infrastructure to the context-aware business process from layer to layer, as well as a solution to deal with the semantic hierarchies of contextual data to ensure the capability and usability of context in the context-aware business process.

Applying the proposed framework on the representative process allows us to identify its benefits and limitations.

- Better fit between IoT implementation and business processes: The proposed framework provides the ways that different layers of context-aware BPM ecosystem can utilize the IoT data. In turn, the IoT data needed for a business process acquiring context-awareness can also provide clues for constructing appropriate IoT infrastructure.

- Good support for leveraging the capability of IoT data in the decision-making of a business process: A separate concern of decisions from the process flow allows the data required to be explicitly presented. These data may involve contextual data and IoT data. Moreover, the context layer further promotes the utilization of IoT data in terms of the semantic hierarchies of contextual information. IoT-integrated context reasoning can obtain up-to-date contextual knowledge for decision making.

- Help for involving dynamic analytics in context-aware BPM: Dynamic analytics need IoT data as its inputs to take external environments and system behaviors into consideration. According to the proposed framework, outcomes of dynamic analytics can get added values by being used for supporting the decision dimension of context-aware business processes as parameters of data requirements or BKMs.

- Limitation in the presentation of utilizing data analytics models: In this representative case, the analytics layer is presented for the logic of its need and utilization in the business process, but is not implemented to depict its capability for optimizing the business process at design time and run time.

- Difficulties in the transformation between business rules and semantics rules for the context reference: Semantic rules for the context reference needs to be consistent with the business logic to ensure the correctness of context interpretation. Up till now, this transformation still relies on expert knowledge without a systematic or automatic way to process.

- Need for the automation of this framework: A tool may be needed to break the barriers between different techniques of layers in the context-aware BPM ecosystem, including ontologies, DMN, data analytics techniques and the BPM technique for obtaining the automation of IoT-enhanced context-aware BPM. 


\section{CONCLUSION AND FUTURE WORK}

Decisions have been considered within the context-aware business process in a traditional way which impairs the capability of contextual information with IoT data involved and data analytics to optimize the business process at both design time and run time. Hence, a separate concern of the decision dimension from the process flow is adopted in this research. After examining the key role that decisions play in context-aware BPM, the context-aware BPM ecosystem is presented which includes business process models, context models, decision models and business process execution. Moreover, IoT data is integrated the context modeling since its capability of capturing the physical world into the digital world. Through the context layer that consists of context vocabulary and context reference, the semantic hierarchies of contextual information can be leveled to ensure the understandability and usability of multi-sources contextual data involving IoT data. This up-to-date contextual knowledge can then be used for inputs of dynamic data analytics or parameters of decision logics and requirements. Outcomes of data analytics can also promote the decision making of business processes.

This work proposes a framework to bridge the gap between the IoT infrastructure and context-aware BPM by integrating IoT data into context ontologies to enhance the decision making of business processes in a methodological way. The connection from layer to layer in the context-aware BPM ecosystem is specifically presented.

In future endeavours we will investigate a complicated case for applying the proposed framework in more detail. In terms of the transformation between context-dependent business rules and semantic rules of context reference, a systematic method is planned to propose for completing the solution. Moreover, the automation of the proposed framework is also interesting for the future research.

\section{ACKNOWLEDGMENT}

This research is supported by the Natural Science Foundation of China under Grant 71502010.

\section{REFERENCES}

[1] C. Perera, A. Zaslavsky, P. Christen, and D. Georgakopoulos, "Context Aware Computing for The Internet of Things: A Survey," IEEE Communications Surveys \& Tutorials, vol. 16, no. 1, pp. 414-454, 2014.

[2] C. Janiesch, A. Koschmider, M. Mecella, B. Weber, A. Burattin, C. Di Ciccio, A. Gal, U. Kannengiesser, F. Mannhardt, J. Mendling, A. Oberweis, M. Reichert, S. Rinderle-Ma, W. Song, J. Su, V. Torres, M. Weidlich, M. Weske, and L. Zhang, The Internet-of-Things Meets Business Process Management: Mutual Benefits and Challenges, arXiv preprint arXiv:1709.03628, 2017.

[3] P. Manlio Del Giudice, M. Ferretti, and F. Schiavone, "Internet of Things and business processes redesign in seaports: The case of Hamburg," Business Process Management Journal, vol. 22, no. 2, pp. 271-284\%@1463-7154,2016.

[4] K. Dar, A. Taherkordi, H. Baraki, F. Eliassen, and K. Geihs, "A resource oriented integration architecture for the Internet of Things: A business process perspective," Pervasive and Mobile Computing, vol. 20, pp. 145-159, 7//, 2015.

[5] S. Meyer, A. Ruppen, and C. Magerkurth, "Internet of Things-Aware Process Modeling: Integrating IoT Devices as Business Process Resources," Advanced Information Systems Engineering: 25th
International Conference, CAiSE 2013, Valencia, Spain, June 17-21, 2013. Proceedings, C. Salinesi, M. C. Norrie and Ó. Pastor, eds., pp. 84-98, Berlin, Heidelberg: Springer Berlin Heidelberg, 2013.

[6] K. Sperner, S. Meyer, and C. Magerkurth, "Introducing Entity-Based Concepts to Business Process Modeling," Business Process Model and Notation: Third International Workshop, BPMN 2011, Lucerne, Switzerland, November 21-22, 2011. Proceedings, R. Dijkman, J. Hofstetter and J. Koehler, eds., pp. 166-171, Berlin, Heidelberg: Springer Berlin Heidelberg, 2011.

[7] J. Taylor, A. Fish, J. Vanthienen, and P. Vincent, "Emerging standards in decision modeling," An introduction to decision model notation, pp. 133-146, 2013.

[8] H.-H. Chiu, and M.-S. Wang, "A Study of IoT-Aware Business Process Modeling," International Journal of Modeling and Optimization, vol. 3, no. 3, pp. 238, 2013.

[9] W. Wang, S. De, R. Toenjes, E. Reetz, and K. Moessner, "A Comprehensive Ontology for Knowledge Representation in the Internet of Things." Security and Privacy in Computing and Communications, pp. 1793-1798, 2012.

[10] D. L. Hall, and J. Llinas, "An introduction to multisensor data fusion," Proceedings of the IEEE, vol. 85, no. 1, pp. 6-23, 1997.

[11] D. Nadoveza, and D. Kiritsis, "Ontology-based approach for context modeling in enterprise applications," Computers in Industry, vol. 65, no. 9, pp. 1218-1231, 2014.

[12] R. R. Oliveira, I. M. G. Cardoso, J. L. V. Barbosa, C. A. da Costa, and M. P. Prado, "An intelligent model for logistics management based on geofencing algorithms and RFID technology," Expert Systems with Applications, vol. 42, no. 15-16, pp. 6082-6097, 2015.

[13] K. Figl, J. Mendling, G. Tokdemir, and J. Vanthienen, "What we know and what we do not know about DMN," Enterprise Modelling and Information Systems Architectures (EMISAJ), vol. 13, pp. 2: 1-16-2: 1-16, 2018.

[14] R. Song, J. Vanthienen, W. Cui, Y. Wang, and L. Huang, "What Roles do Decisions Play in Context-Aware Business Process Management?," Accepted for Proceedings of the 21st International Conference on Enterprise Information Systems (ICEIS 2019), 2019.

[15] B. Heinrich, and D. Schön, "Automated planning of context-aware process models," Proceedings of the European Conference on Infromation Systems, Germany, 2015.

[16] G. Regev, I. Bider, and A. Wegmann, "Defining business process flexibility with the help of invariants," Software Process: Improvement and Practice, vol. 12, no. 1, pp. 65-79, 2007.

[17] F. Tang, M. Guo, M. Dong, M. Li, and H. Guan, "Towards ContextAware Workflow Management for Ubiquitous Computing," 2008 International Conference on Embedded Software and Systems. IEEE, pp. 221-228, 2008.

[18] Z. Jaroucheh, X. Liu, and S. Smith, "Apto: a MDD-based generic framework for context-aware deeply adaptive service-based processes." 2010 IEEE International Conference on Web Services. p p. 219-226, 2010.

[19] J. Taylor, J. Purchase, and R. M. Soley, Real-world decision modeling with DMN: Meghan-Kiffer Press Tampa, 2016.

[20] R. Vidgen, and X. Wang, "From Business Process Management to Business Process Ecosystem," Journal of Information Technology, vol. 21, no. 4, pp. 262-271, 2006/12/01, 2006.

[21] G. D. Abowd, A. K. Dey, P. J. Brown, N. Davies, M. Smith, and P. Steggles, "Towards a better understanding of context and contextawareness." International symposium on handheld and ubiquitous computing.p p. 304-307, 1999.

[22] J. Ye, L. Coyle, S. Dobson, and P. Nixon, "Ontology-based models in pervasive computing systems," The Knowledge Engineering Review, vol. 22, no. 4, pp. 315-347, 2007.

[23] E. Serral, J. De Smedt, M. Snoeck, and J. Vanthienen, "Contextadaptive Petri nets: Supporting adaptation for the execution context," Expert Systems with Applications, vol. 42, no. 23, pp. 9307-9317, 2015. 